\title{
SINGLE-CRYSTAL NEUTRON DIFFRACTION
}

Thomas F. Koetzle

Chemistry Department, Brookhaven National Laboratory, P.O. Box 5000, Upton, NY 11973 and

Intense Pulsed Neutron Source, Argonne National Laboratory, Argonne, IL 60439

Telephone: (631) 344-4384

Fax: (631) 344-5815

E-mail: tkoetzle@aol.com

Diskette Type: Microsoft Word for Office 2000

File Name: Wiley_Unit.doc 


\section{INTRODUCTION}

Single-crystal neutron diffraction measures the elastic Bragg reflection intensities from crystals of a material, the structure of which is the subject of investigation. A single crystal is placed in a beam of neutrons produced at a nuclear reactor or at a proton accelerator-based spallation source. Single-crystal diffraction measurements are commonly made at thermal neutron beam energies, which correspond to neutron wavelengths in the neighborhood of 1 Angstrom. For high-resolution studies requiring shorter wavelengths (ca. $0.3-0.8$ Ångstroms), a pulsed spallation source or a hightemperature moderator (a "hot source") at a reactor may be used. When complex structures with large unit-cell repeats are under investigation, as is the case in structural biology, a cryogenic-temperature moderator (a "cold source") may be employed to obtain longer neutron wavelengths ( $c a .4-10$ Angstroms). ${ }^{1}$

A single-crystal neutron diffraction analysis will determine the crystal structure of the material, typically including its unit cell and space group, the positions of the atomic nuclei and their mean-square displacements, and relevant site occupancies. Because the

\footnotetext{
${ }^{1} \mathrm{~A}$ monochromatic beam technique is generally used at reactors, which normally operate as $\mathrm{CW}$ neutron sources. At spallation sources, which normally are pulsed, a time-of-flight Laue method with a broad range of neutron wavelengths is generally used, and the neutron wavelength is determined from the time-of-flight for neutrons to reach the detector. The type of moderator is again generally optimized for the particular application, similar to the common practice at reactors.
} 
neutron possesses a magnetic moment, the magnetic structure of the material can be determined as well, from the magnetic contribution to the Bragg intensities. This latter aspect falls beyond the scope of the present unit; for information on magnetic scattering of neutrons see Unit 14.3.

Instruments for single-crystal diffraction (single-crystal diffractometers or SCDs) are generally available at the major neutron scattering center facilities. Beam time on many of these instruments is available through a proposal mechanism. A listing of neutron SCD instruments and their corresponding facility contacts is included in an appendix accompanying this unit.

Complementary and Related Techniques

A number of techniques give information on crystal structure that is complementary to that provided by single-crystal neutron diffraction. Some of the most important of these are listed below along with a brief description. For additional information on these techniques, and their advantages and disadvantages, the reader is referred to the corresponding units of this manual in which they are described. Here at the outset, it is worth noting that neutron diffraction is a very expensive proposition, first of all involving the often demanding and time-consuming preparation of large, highquality crystals (at least $1 \mathrm{~mm}^{3}$ in volume). Then it is generally necessary to travel to a neutron scattering research center to carry out the measurements, which themselves typically require from several days to several weeks to complete. Readers will therefore 
want to be cerlain that neutron diffraction is the technique of choice for solving their problem at hand - otherwise one of the complementary methods certainly should be considered first.

Single-crystal x-ray diffraction (Unit 11.1) is the method most directly complementary to single-crystal neutron diffraction. The chief difference between the two methods is that, while neutron diffraction images the nuclear scattering density in the crystal, $\mathrm{x}$-ray diffraction images the electron-density distribution. Neutron diffraction therefore has important advantages in studies where hydrogen or other light atoms must be located, or where isotopic substitutions may be of interest, and of course for magnetic structures. ${ }^{2}$ X-ray and neutron diffraction measurements can be combined to good effect in studies seeking detailed information about the valence electron-density distribution in a material, particularly when hydrogen is present (Coppens, 1997).

Powder neutron and powder x-ray diffraction are also powerful techniques for determining crystal structure. Of course, for many important classes of materials single crystals may be difficult, or even impossible to obtain. Powder diffraction methods have the advantage of wide applicability and generally allow for much more rapid data collection than is the case for single-crystal methods. This makes powder methods ideal for studies of materials over ranges of temperature and pressure, for example in studies

\footnotetext{
${ }^{2}$ Magnetic structures can also be studied using $\mathrm{x}$ rays, although there the effect is much smaller so that synchrotron radiation is generally required (see Unit 11.3). In the special case where the $\mathrm{x}$-ray energy is close to an absorption edge of a magnetic center in the sample, resonance enhancement of the magnetic scattering can produce effects comparable in magnitude to those observed with neutrons.
} 
investigating phase transitions and for in situ, real-time studies. The chief disadvantage of powder methods stems from the more limited amount of information obtained in the diffraction pattern, which generally places an upper limit on the complexity of structures that can satisfactorily be treated. Progress in this regard continues at a rapid pace, however, so that structures with on the order of 100 independent atoms in the crystallographic asymmetric unit may now be considered as suitable candidates for powder studies. Recently, powder diffraction studies of proteins have even been undertaken, fitting highly constrained structure models to the data (Von Dreele, 1999). Both powder and single-crystal x-ray techniques have been enormously enhanced by the availability of synchrotron $\mathrm{x}$-ray sources, with their extremely high-intensity beams. At the newest, third-generation synchrotron sources, $x$-ray intensities may be as much as nine or ten orders of magnitude larger than for the most intense neutron beams that are currently available.

Electron diffraction (Chapter 12b) is another complementary technique for crystal-structure determination, particularly for example when studying surfaces and interfaces where low-energy electron diffraction (Unit 12b.1) is especially powerful, or when only micro crystals are available. Progress in n-beam dynamical diffraction computations is extending the power of electron diffraction for the solution and refinement of crystal structures.

Nuclear magnetic resonance (Unit 10a.1) is also a valuable technique for investigating the structure of materials. To cite just one application, high-field solid-state 
NMR can yield accurate distances between hydrogen atoms and, particularly in materials with high thermal motion or disorder, results may sometimes be more reliable than for comparable interatomic distances obtained from neutron diffraction.

Scope of This Unit

This unit outlines the basic principles of single-crystal neutron diffraction and provides examples to illustrate the range of materials that can be studied. Sufficient practical details are included, it is hoped, to help a non-expert to get started with the technique and to locate an appropriate neutron diffraction facility. A listing of the instruments that are available at various neutron sources is included as appendix material.

For readers desiring additional background, the classic monograph by Bacon (1975) provides an excellent starting point. Wilson (1999) has recently published an overview that includes a comprehensive survey of neutron diffraction results on molecular materials. For those wishing to investigate the subject in more detail, Marshall and Lovesey (1971) provide an exhaustive theoretical treatment of all aspects of neutron scattering.

\section{PRINCIPLES OF THE METHOD}

Single-crystal neutron diffraction measures elastic, coherent scattering, i.e., the Bragg reflection intensities. Although the measurements and their interpretation are 
generally quite straightforward, and amount to a standard crystal-structure analysis analogous to what is done with $\mathrm{x}$ rays, neutron structure determinations are still relatively uncommon. This is so, because of the limited number of neutron crystallography facilities and the need to obtain large samples in order to observe adequate reflection intensities (vide supra). Thus, for example, the contents of a recent release of the Cambridge Structural Database of organic and organometallic crystal structures (Allen and Kennard, 1993) consist of less than $1 \%$ neutron structures (the overwhelming majority of the remaining $99 \%$ are $\mathrm{x}$-ray structures).

Basic Theory of Single-Crystal Neutron Diffraction

The nuclear scattering density in a crystal may be expressed in terms of the neutron structure factors, $F(h, k, l)$, by the familiar relationship

$$
\rho(x, y, z)=\Sigma F(h, k, l) \exp \{-2 \pi \mathrm{i}(\mathrm{hx}+\mathrm{ky}+\mathrm{lz})\}
$$

where the Fourier summation (1) runs over the Miller indices $h, k, l$, and the coordinates $\mathrm{x}, \mathrm{y}, \mathrm{z}$ are expressed in terms of fractions of the unit-cell translations $\mathbf{a}, \mathbf{b}, \mathbf{c}$. The nuclear scattering density is simply the atomic probability density at any point multiplied by the corresponding neutron scattering amplitude.

A principal difference between neutron and $\mathrm{x}$-ray diffraction derives from the fact that neutrons are scattered by the atomic nuclei in a crystal, while $\mathrm{x}$ rays are scattered by the electrons. Because the nuclear radius is approximately $10^{4}$ times smaller than the neutron wavelengths that are used in diffraction, the neutron scattering amplitude is a 
constant independent of the momentum transfer, $Q=4 \pi(\sin \theta / \lambda)$. This contrasts with the situation for $\mathrm{x}$ rays where the scattering amplitude, also called the scattering factor or form factor, falls off with increasing $Q$ due to the destructive interference of the scattering from different regions of the electron-density cloud. Each isotope has a unique neutron scattering amplitude, but these values lie within the same order of magnitude across the entire periodic table as is shown in Figure $1 .^{3}$ For this reason neutron diffraction has important advantages in studies where hydrogen or other light atoms must be located, or where isotopic substitutions may be of interest, as has been mentioned earlier. Further, because neutron diffraction images the nuclear scattering density in the crystal, it provides an unbiased estimate of the mean nuclear position. In contrast, x-ray diffraction studies generally assume that the nucleus is located at the centroid of its electron-density cloud. This can be a poor assumption, e.g., where hydrogen atoms form covalent bonds. ${ }^{4}$

It is of course not possible to obtain the scattering-density function $\rho(x, y, z)$ directly from the observed diffraction intensities, because of the crystallographic phase problem. In practice, in most cases an initial estimate of the neutron structure factor phases is obtained from a prior $x$-ray diffraction study. 'Probabilistic direct methods of phase determination developed for X-ray structure determination have however also been shown to work in the neutron case, e.g., for organic (Verbist et al., 1972) and

\footnotetext{
${ }^{3}$ For $\mathrm{x}$ rays, the scattering amplitude increases with the number of atoms so that the diffraction is dominated by contributions from the high- $\mathrm{Z}$ atoms.
} 
organometallic crystals (Broach et al., 1979). Direct methods seem to work in practice in spite of the existence of negative scattering density whenever hydrogen is present, which actually contradicts one of the underlying assumptions of direct methods. Phasedetermination methods based on anomalous dispersion can also be applied in neutron diffraction, for special cases involving crystals that contain one of a handful of highly absorbing elements, including $\mathrm{Li}, \mathrm{B}, \mathrm{Cd}, \mathrm{Sm}$, and $\mathrm{Gd}$ (see, e.g., Koetzle and Hamilton, 1975, and references cited therein).

Neutron structure analysis and refinement generally utilizes standard kinematical diffraction theory. According to kinematical theory, the Bragg reflection intensity $\mathrm{I}(\mathrm{h}, \mathrm{k}, \mathrm{l})$ is given by the relationship 5

$$
\mathrm{I}(\mathrm{h}, \mathrm{k}, \mathrm{l})=\left(\mathrm{k} \lambda^{2} \mathrm{~V}_{\mathrm{d}} / \mathrm{V}^{2}\right)|\mathrm{F}(\mathrm{h}, \mathrm{k}, \mathrm{l})|^{2}
$$

where $\quad k=a$ scale factor proportional to the neutron beam intensity,

$$
\begin{aligned}
& \mathrm{V}_{\mathrm{c}}=\text { the crystal volume, } \\
& \mathrm{V}=\text { the unit-cell volume, } \\
& \text { and }|\mathrm{F}(\mathrm{h}, \mathrm{k}, \mathrm{l})|^{2}=\text { the squared structure factor. }
\end{aligned}
$$

The structure factor is

$$
\mathrm{F}(\mathrm{h}, \mathrm{k}, \mathrm{l})=\Sigma \mathrm{a}_{\mathrm{i}} \mathrm{b}_{\mathrm{i}} \exp \left(-\mathrm{W}_{\mathrm{i}}\right) \exp \{2 \pi \mathrm{i}(\mathrm{hx}+\mathrm{ky}+\mathrm{lz})\}
$$

${ }^{4}$ For example, $\mathrm{C}-\mathrm{H}$ bond distances determined by $\mathrm{x}$-ray diffraction are systematically shortened by $\mathrm{ca} .0 .1$ $\AA$ due to the effects of bonding on the hydrogen 1s electron-density distribution.

${ }^{5}$ For monochromatic beam methods, the scale factor $\mathrm{k}$ is normally a constant. For Laue methods, $\mathrm{k}$ is a function of the neutron wavelength reflecting the neutron flux spectrum incident on the sample. Equation (2) omits the Lorenz term, which must be introduced to correct for the geometrical effect caused by scanning reflections to obtain integrated Bragg intensities. 
where

$$
\begin{aligned}
& a_{i}=\text { the atomic site occupancy, } \\
& b_{i}=\text { the neutron scattering amplitude, } \\
& \text { and } W_{i}=\text { the Debye-Waller factor, }
\end{aligned}
$$

and the summation in (3) runs over $\mathrm{n}$ atoms in the crystallographic unit cell.

From (2) and (3), it is apparent that the diffracted intensity will fall off as the unitcell volume increases. In order to achieve sufficient diffracted intensity it is therefore necessary to grow larger single crystals for materials with larger unit cells. The use of longer neutron wavelengths is also highly desirable in this case, e.g., by performing the experiment at an instrument located on a cold moderator bcamline.

The Debye-Waller factor $\mathrm{W}_{\mathrm{i}}$ takes into account the reduction in diffracted intensity from interference produced by smearing of the scattering density due to thermal motion in the sample. In the isotropic approximation

$$
\mathrm{W}_{\mathrm{i}}=\exp \left\{-\mathrm{U}_{\mathrm{i}} \mathrm{Q}^{2} / 2\right\}=\exp \left\{-8 \pi^{2} \mathrm{U}_{\mathrm{i}}\left(\sin ^{2} \theta / \lambda^{2}\right)\right\}
$$

where $U_{i}=$ the atomic mean-square displacement $(\mathrm{adp}){ }^{6}$

Neutron diffraction is often the method of choice in studies aiming at a precise description of thermal motion and/or disorder. This is so, because it is easier to determine the Debye-Waller factor in the neutron case where the atomic scattering

\footnotetext{
${ }^{6}$ It is often the practice to introduce an anisotropic harmonic model in which second-order tensors are employed to describe the adp's. In that case, the Debye-Waller factor is given by

$$
W_{i}=\exp \left\{-2 \pi^{2}\left(U_{11} a^{*^{2}} h^{2}+U_{22} b^{* 2} k^{2}+U_{33} c^{* 2} l^{2}+2 U_{12} a^{*} \cdot b^{*} h k+2 U_{13} a^{*} \cdot c^{*} h l+2 U_{23} b^{*} \cdot c^{*} k l\right\} .\right.
$$
}

Higher-order tensor descriptions may be used to model anharmonic effects. 
amplitude is a constant independent of $\mathrm{Q}$ (vide supra), and there is therefore minimal correlation between the Debye-Waller factor and the site occupancy. In contrast, the xray atomic form factor may assume a $\mathrm{Q}$ dependence quite similar to that of the DebyeWaller factor, leading to high correlations in the least-squares structure refinement.

The fact that the neutron scattering amplitude is independent of Q confers an additional advantage that is perhaps not always well appreciated. When the diffraction measurements are made at cryogenic temperatures, lowering the Debye-Waller factors, neutron Bragg intensities will exhibit a much-reduced fall off with $Q$ compared to the $x$ ray case. This confers substantial benefits in terms of improved precision in the neutron atomic positions.

The standard kinematical theory outlined above ignores the effects of absorption and extinction in real crystals. In practice, absorption is often quite minimal except in the case of crystals that contain hydrogen where the effective absorption due to hydrogen incoherent scattering can be quite appreciable. When desired, the measured neutron intensities can be corrected for absorption using the method of Gaussian integration (Busing and Levy, 1957) or employing an analytical technique in which the crystal is described in terms of Vornoi polyhedra (Templeton and Templeton, 1973).

Because of the requirement for large crystals, extinction corrections are often required during neutron structure refinement in order to obtain a satisfactory fit to the strong reflections. A number of formalisms have been developed for this purpose with 
perhaps the most widely used being that due to Becker and Coppens (1975), which in its most general implementation treats both primary and secondary extinction.

PRACTICAL ASPECTS OF THE METHOD

The initial applications of single-crystal neutron diffraction to the study of molecular structure were carried out at the first generation of research reactors around 1950 (see, e.g., the studies at Oak Ridge National Laboratory of $\mathrm{KHF}_{2}$ (Peterson and Levy, 1952) and ice (Peterson and Levy, 1953, 1957) and the study at Harwell ${ }^{7}$ of $\mathrm{KH}_{2} \mathrm{PO}_{4}, \mathrm{KDP}$ (Bacon and Pease, 1953, 1955). These studies provided an important demonstration of the advantages of neutron diffraction for determining the positions of hydrogen atoms and studying hydrogen bonding.

Single-crystal methods are being employed at modern reactor and spallation neutron sources to study the structures of a broad range of materials. For example, the tin hydride complex shown in Figure 2 was investigated (Hẹning et al., 2000) using the SCD spectrometer at the Intense Pulsed Neutron Source (IPNS) at Argonne National Laboratory. Collection of Bragg intensity data at a temperature of $20 \mathrm{~K}$ for this triclinic crystal with 111 independent atoms, unit-cell volume $V=1986 \AA^{3}$, and crystal volume $V_{c}=15 \mathrm{~mm}^{3}$ required eleven days on SCD. Low-temperature studies of this quality allow full, unconstrained refinement of the structure model incorporating anisotropic Debye-Waller factors and readily yield metal-hydrogen bond distances with a precision

\footnotetext{
${ }^{7} \mathrm{KDP}$ was also studied independently at about the same time at Oak Ridge (Peterson et al., 1953, 1954).
} 
of better than $0.01 \AA$. The amount of beam time used by this experiment is quite representative at present at IPNS for this type of organometallic crystal.

Figure 3 shows an application of Fourier methods to single-crystal neutron diffraction data obtained at the Brookhaven National Laboratory High Flux Beam Reactor (HFBR). ${ }^{8}$ In this site-specific isotope labeling study of a chiral organic alcohol (Yuan et al., 1994) the neutron Fourier map readily gives the location of the deuterium label that was introduced in an enzymatic hydrogenation reaction.

Figure 4 shows the results of a study of the variation with temperature of the anharmonic thermal motion in the cubic form of $\mathrm{ZnS}$, zincblende (Moss, McMullan and Koetzle, 1980, 1983). This high-resolution study, also carried out on one of the singlecrystal diffractometers at the HFBR, used a relatively short neutron wavelength of $0.83 \AA$ and successfully modeled the higher-order contributions to the Debye-Waller factor for a zincblende sample drawn from the collections of the Smithsonian Institution, Washington, DC, U.S.A.

\section{Neutron Sources and Instrumentation}

As mentioned earlier, neutron sources fall into two categories: nuclear reactors, which normally operate as $\mathrm{CW}$ neutron sources, and spallation sources, which normally

\footnotetext{
${ }^{8}$ The HFBR neutron source ceased operation in 1999.
} 
are pulsed. ${ }^{9}$ A listing of neutron scattering centers offering single-crystal diffraction facilities that will host users from other organizations is given in the appendix accompanying this unit.

Figure 5 schematically illustrates a representative SCD set-up at a reactor. Using this facility, which was formerly installed on a dual thermal beam port at the HFBR at Brookhaven, neutron beams in the wavelength range $0.8-1.7 \AA$ could be extracted using a variety of monochromator crystals. ${ }^{10}$ Samples were mounted on one of the two fourcircle diffractometers with Eulerian cradlcs that provided for full orientation control. Integrated Bragg intensities were recorded by scanning the samples in steps about the vertical $(\omega)$ axis, counting for a preset incident-beam monitor count to correct for any variation in the incident neutron flux from the reactor over time. The addition of position-sensitive area detectors has significantly enhanced the performance of many of the SCDs currently in operation at reactors (vide infra). In particular, the recent emergence of neutron imaging-plate detectors and the development of improved glass scintillation detectors have been important advances.

Figure 6 is a schematic view of the SCD at Argonne's IPNS, which serves as an illustration of an instrument at a spallation neutron source. This diffractometer (Schultz, 1987), which is installed at a beam port on a liquid methane cryogenic moderator,

\footnotetext{
${ }^{9}$ A pulsed nuclear reactor (IBR-30) operates at The Franck Laboratory of Nuclear Physics in Dubna, Russia, and a CW spallation source (SINQ) operates at the Paul Scherer Institute in Villigen, Switzerland. ${ }^{10}$ Common choices for monochromator crystals include $\mathrm{Be}, \mathrm{Si}, \mathrm{Ge}, \mathrm{Cu}$, and pyrolitic graphite.
} 
operates by the time-of-flight Laue method. The full spectrum of neutrons from the moderator is allowed to impinge on the sample, and the wavelength is determined by the time-of-flight required for neutrons to reach the detector, viz.,

$$
\lambda=\mathrm{ht} / \mathrm{md}
$$

where

$$
\begin{aligned}
& \mathrm{h}=\text { Planck's constant, } \\
& \mathrm{t}=\text { the neutron time-of-flight, } \\
& \mathrm{m}=\text { the neutron mass, } \\
& \text { and } \mathrm{d}=\text { the source-to-detector distance. }
\end{aligned}
$$

The Eulerian cradle mount is used to orient the sample for a series of intensity data collection histograms, recording $t$ and the position for each neutron arriving at the area detector. Counts are accumulated for a preset number of accelerator pulses. ${ }^{11}$ With this geometry, a complete hemisphere of reciprocal space can be explored while collecting a total of 45 data histograms. In contrast to monochromatic beam methods, where the sample must be rotated to scan through Bragg reflections in order to obtain integrated intensities, in the Laue method the sample is held stationary while counting is in progress. This significantly simplifies the experiment and may be of particular advantage, for example when a special sample environment is required or when carrying out rapid reciprocal-lattice surveys, e.g., while searching for phase transitions over a range of temperature and/or pressure.

\footnotetext{
${ }^{11}$ The IPNS proton accelerator operates at $30 \mathrm{~Hz}$ and a proton beam power of $7 \mathrm{~kW}$. A depleted uranium spallation target is employed at present.
} 
In general, each neutron scattering center has developed its own instrumentation, and the design of each SCD spectrometer is unique in its details. From the prospective experimenter's point of view, however, most SCDs probably will look quite similar in the sense that they will feature an Eulerian cradle sample mount that offers the possibility of deploying cryostats, furnaces, pressure cells, etc., to obtain a wide range of sample environments. As was mentioned earlier, a successful experiment requires production of a large, well-formed single crystal. Generally, the minimum satisfactory sample size falls in the range 1-10 $\mathrm{mm}^{3}$, with larger samples required for larger unit cells. These large samples are required because even at the current most powerful spallation neutron source, the $180 \mathrm{~kW}$ ISIS at the Rutherford Appleton Laboratory in the U.K., or at the most powerful high-flux reactor sources, the $58 \mathrm{MW}$ High Flux Reactor (HFR) at the Institut Laue-Langevin in Grenoble, France and the $85 \mathrm{MW}$ High Flux Isotope Reactor (HFIR) at Oak Ridge National Laboratory in the U.S.A., the neutron flux on sample at the SCDs is several orders of magnitude reduced compared to the flux from a conventional, sealedtube laboratory x-ray source.

Bent crystal focusing monochromators (see, e.g., Tanaka et al., 1999b, 2000) have been installed at a number of reactor SCD instruments. Significant intensity enhancement has been achieved in this way, in some cases approaching one order of magnitude. 
Detectors

The first generation of SCD instruments at reactors utilized single-channel gas proportional counters filled with $\mathrm{BF}_{4}$ or ${ }^{3} \mathrm{He}$. These detectors capture neutrons by the reactions ${ }^{10} \mathrm{~B}+{ }^{1} \mathrm{n}={ }^{7} \mathrm{Li}+{ }^{4} \mathrm{He}+2.8 \mathrm{MeV}$, and ${ }^{3} \mathrm{He}+{ }^{1} \mathrm{n}={ }^{1} \mathrm{H}+{ }^{3} \mathrm{H}+0.76 \mathrm{MeV}$, respectively. The thermal neutron capture cross section of ${ }^{3} \mathrm{He}$ is about 1.5 times that of ${ }^{10} \mathrm{~B}$, and for this reason ${ }^{3} \mathrm{He}$ counters are usually lighter and smaller than $\mathrm{BF}_{4}$ counters.

Modern neutron SCD instruments generally include two-dimensional positionsensitive detectors, allowing for parallel data collection. Area detectors are, in any event, required for the time-of-flight Laue instruments'at pulsed spallation sources. Neutron position-sensitive detectors are of three types: gas-filled proportional counters, generally using ${ }^{3} \mathrm{He}$; scintillation counters, such as the ${ }^{6} \mathrm{Li}$-glass detector on the SCD at IPNS (see Figure 6); and imaging-plate detectors, which utilize a Gd converter. Neutron imaging plates (Niimura et al., 1994, Tazaki et al., 1999) have the important advantage of being flexible, which facilitates covering a large solid angle. Powerful new SCDs utilizing imaging plates and oscillation/Weissenberg geometry have been developed since 1990 (LADI, Wilkinson and Lehmann, 1991, Cipriani et al., 1995; BDX, Tanaka et al, 1999a). Imaging plates are integrating detectors and are therefore unfortunately not suitable for use on time-of-flight Laue instruments. 


\section{DATA ANALYSIS AND INITIAL INTERPRETATION}

As discussed in the section of this unit on theory, initial values of the neutron structure factor phases are usually calculated based on a structure model obtained from a prior x-ray diffraction study. Although the neutron phases can be obtained by direct methods, vide supra, the $\mathrm{x}$-ray study provides an efficient structure solution and serves the important dual purpose of allowing a check for problems such as twinning that might make it impossible to obtain a high-quality neutron structure. Most neutron scattering facilities strongly recommend that prospective SCD users have an X-ray structure of their material in hand before embarking on their neutron measurements.

Each SCD instrument generally provides its users with its own specially adapted suite of programs to handle data reduction, i.e., for Bragg peak indexing, integration of peak intensities, absorption correction, and merging of equivalent reflections. ${ }^{12}$ For information on this software the reader should consult the facility contact websites that are listed in the Appendix. These websites generally identify the instrument responsible scientists who can advise prospective users on what to expect.

Refinement of neutron structures may be carried out with a standard crystallographic program package. Program systems in common use at neutron scattering centers include GSAS (Larson and Von Dreele, 1999) and SHEL-X (Sheldrick, 1993).

\footnotetext{
${ }^{12}$ For Laue data, equivalent reflections are generally not merged, since they may be collected at different neutron wavelengths and their extinction corrections may therefore differ one from another.
} 


\section{SAMPLE PREPARATION}

As has been noted above, single-crystal neutron diffraction requires a large, highquality crystal, generally at least $1 \mathrm{~mm}^{3}$ in volume. The sample is normally mounted on an aluminum or vanadium ${ }^{13}$ pin with a suitable adhesive. The facility's SCD instrument responsible can advise the user as to the proper type of pin to use. Often the most suitable course will be to bring samples to the neutron scattering center unmounted, and to use the local laboratory facilities for final sample preparation.

The availability of appropriate on-site sample-handling facilities is frequently an important factor to consider when choosing a neutron scattering center. For example, prospective users with air-sensitive samples will want to be certain that facilities exist for handling and mounting their crystals under an inert atmosphere. The availability of wellequipped conventional laboratory facilities adjacent to the neutron source can critically affect the outcome of an experiment.

\section{PROBLEMS}

The application of neutron scattering in general, including single-crystal diffraction, has always been constrained by the limited number of research facilities 
available. With the advent of the 2 MW Spallation Neutron Source (SNS), now under construction at Oak Ridge and scheduled to begin operation in 2006 , the neutron scattering community will have at its disposal a state-of-the-art facility of unprecedented power and intensity, with approximately an order of magnitude increase over the power currently delivered by ISIS. ${ }^{14}$ The SNS is expected to make possible a broad range of new SCD applications. For example, it should be possible to:

1. Use much smaller single crystals, perhaps approaching normal x-ray sized samples (ca. $0.01 \mathrm{~mm}^{3}$ in volume), which will greatly expand the range of materials that can be investigated.

2. Obtain complete SCD data sets in minutes, as opposed to the days or weeks that are presently required. This should enable the study of many interesting processes in real time. To cite just one interesting example, it should be possible to investigate solid-state photochemical reactions where the mechanisms can be probed by following site-specific isotope labels (Ohgo et al., 1996, 1997).

3. Carry out extensive parametric studies including investigating materials over a broad range of temperatures and pressures, which in practice can only be done conveniently with powders at existing facilities. This would be extremely important for many materials applications, e.g., to understand the response of

\footnotetext{
${ }^{13}$ Vanadium is sometimes used to reduce the background because of its very small neutron scattering amplitude. Aluminum will usually suffice, however, so long as the diameter of the pin is substantially smaller than that of the crystal.

${ }^{14}$ Planning for major new spallation facilities is at an advanced stage in Europe, with the European Spallation Source (ESS) project, and also in Japan (JHF project).
} 
hydrogen-bonded ferroelectric, ferroelastic and non-liner optical (NLO) materials to temperature and pressure changes.

A critical challenge facing the neutron scattering community is how to replace the facilities at the current generation of research reactors. The majority of these facilities were commissioned in the 1950's and 1960's, and some are nearing the end of their projected lifetimes. Two new research reactors are currently under construction, at Garching, Germany, where the 20 MW FRM-II is scheduled to be completed in 2001, and at ANSTO, Lucas Heights, Australia, where a $20 \mathrm{MW}$ replacement for the HIFAR reactor is scheduled to completed in 2005 .

\section{ACKNOWLEDGEMENTS}

The author would like to thank Dr. A.J. Schultz for reading a draft of this manuscript and providing comments. Work at Argonne and Brookhaven National Laboratories was supported by the U.S. Department of Energy, Office of Basic Energy Sciences, under Contracts W-31-109-ENG-38 and DE-AC2-98CH10886, respectively. Additional financial support was provided to the author by NATO under grant PST.CLG.976225. 


\section{LITERATURE CITED}

Allen, F.H. and Kennard, O. 1993. 3D Search and Research Using the Cambridge Structural Database. Chem. Des. Autom. News 8(1):31-37.

Bacon, G.E. 1975. Neutron Diffraction (Third Edition). Oxford University Press, London.

Bacon, G.E. and Pease, R.S. 1953. A Neutron Diffraction Study of Potassium Dihydrogen Phosphate by Fourier Synthesis. Proc. Roy. Soc. A220:397-421.

Bacon, G.E. and Pease, R.S. 1955. A Neutron-Diffraction Study of the Ferroelectric Transition of Potassium Dihydrogen Phosphate. Proc. Roy. Soc. A230:359-381.

Becker, P.J. and Coppens, P. 1975. Extinction within the Limit of Validity of the Darwin Transfer Equations. III. Non Spherical Crystals and Anisotropy of Extinction. Acta Crystallogr. A31:417-425.

Broach, R.W., Schultz, A.J., Williams, J.M., Brown, G.M., Manriquez, J.M., Fagan, P.J., and Marks, T.J. 1979. Molecular Structure of an Unusual Organoactinide Hydride Complex Determined Solely by Neutron Diffraction. Science 203:172-174. 
Busing, W.R. and Levy, H.A. 1957. High Speed Computation of the Absorption Correction for Single Crystal Diffraction Measurements. Acta Crystallogr. 10:180187.

Cipriani, F., Castagna, J.-C., Lehmann, M.S., and Wilkinson, C. (1995). A Large ImagePlate Detector for Neutrons. Physica B 213-214:975-977.

Coppens, P. 1997. X-ray Charge Densities and Chemical Bonding. Oxford University Press.

Henning, R.A., Albinati, A., Klooster, W.T., Power, P., and Schultz, A.J. 2000. SingleCrystal Neutron Diffraction Study of a Tin(II) Hydride Complex. To be published.

Koetzle, T.F. and Hamilton, W.C. 1975. Neutron Diffraction Study of NaSmEDTA. $8 \mathrm{H}_{2} \mathrm{O}$ : An Evaluation of Methods of Phase Determination Based on Three-Wavelength Anomalous Dispersion Data. In Anomalous Scattering (S. Ramaseshan and S.C. Abrahams, eds.) pp. 489-502. Munksgaard, Copenhagen.

Larson, A.C. and Von Dreele, R.B. 1999. GSAS General Structure and Analysis System. Los Alamos National Laboratory, New Mexico, U.S.A.

Marshall, G.W., and Lovesey, S.W. 1971. Theory of Thermal Neutron Scattering. Oxford University Press, London. 
Moss, B., McMullan, R.K., and Koetzle, T.F. 1980. Temperature Dependence of Thermal Vibrations in Cubic ZnS: A Comparison of Anharmonic Models. J. Chem. Phys. 73:495-508.

Moss, B., Roberts, R.B., McMullan, R.K., and Koetzle, T.F. 1983. Comment on “Temperature Dependence of Thermal Vibrations in Cubic ZnS: A Comparison of Anharmonic Models". J. Chem. Phys. 78:7503-7505.

Niimura, N., Karasawa, Y., Tanaka, I., Miyahara, J., Takahashi, K., Saito, H., Koizumi, S., and Hidaku, M. An Imaging Plate Neutron Detector. Nucl. Instr. Meth. Phys. Res. A349:521-525.

Ohgo, Y., Ohashi, Y., Klooster, W.T., and Koetzle, T.F. 1996. Direct Observation of Hydrogen-Deuterium Exchange Reaction in a Cobaloxime Crystal by Neutron Diffraction. Chem. Lett. 445-446, 579.

Ohgo, Y., Ohashi, Y., Klooster, W.T., and Koetzle, T.F. 1997. Analysis of HydrogenDeuterium Exchange Reaction in a Crystal by Neutron Diffraction. Enantiomer $2: 241-248$. 
Peterson, S.W. and Levy, H.A. 1952. A Single Crystal Neutron Diffraction

Determination of the Hydrogen Position in Potassium Bifluoride. J. Chem. Phys. 20:704-707.

Peterson, S.W. and Levy, H.A. 1953. A Single-Crystal Neutron Diffraction Study of Heavy Ice. Phys. Rev. 92:1082.

Peterson, S.W. and Levy, H.A. 1957. A Single-Crystal Neutron Diffraction Study of Heavy Ice. Acta Crystallogr. 10:70-76,344.

Peterson, S.W., Levy, H.A., and Simonsen, S.H. 1953. Neutron Diffraction Study of Tetragonal Potassium Dihydrogen Phosphate. J. Chem. Phys. 21:2084-2085.

Peterson, S.W., Levy, H.A., and Simonsen, S.H. 1954. Neutron Diffraction Study of the Ferroelectric Modification of Potassium Dihydrogen Phosphate. Phys. Rev. 93: 11201121.

Schultz, A.J. 1987. Pulsed Neutron Single-Crystal Diffraction. Trans. Am. Crystallogr. Assoc. 23:61-69.

Sheldrick, G.M. 1993. SHELXL-93. Program for the Refinement of Crystal Structures using Single Crystal Diffraction Data. University of Göttingen, Germany. 
Tanaka, I., Ahmed, F.U., and Niimura, N. 2000. Application of a Stacked Elastically Bent Perfect Si Monochromator with Identical and Different Crystallographic Planes for Single Crystal and Powder Neutron Diffractometry. Physica B 283:195-298.

Tanaka, I., Kurihara, K., Haga, Y., Minezaki, Y., Fujiwara, S., Kumazawa, S., and Niimura, N. 1999a. An Upgraded Neutron Diffractometer (BIX-I $\mathrm{I}_{\mathrm{M}}$ ) for Macromolecules with a Neutron Imaging Plate. J. Phys. Chem. Solids 60:1623-1626.

Tanaka, I., Niimura, N., and Mikula, P. 1999b. An Elastically Bent Silicon Monochromator for a Neutron Diffractometer. J. Appl. Crystallogr. 32:525-529.

Tazaki, S., Neriishi, K., Takahashi, K., Etoh, M., Karasawa, Y., Kumazawa, S., and Niimura, N. 1999. Development of a New Type of Imaging Plate for Neutron Detection. Nucl. Instr. Meth. Phys. Res. A424:20-25.

Templeton, D.H. and Templeton, L.K. 1973. Am. Crystallogr. Assoc. Meeting Abstracts, Abstract E10, Storrs, Connecticut, U.S.A.

Von Dreele, R.B. 1999. Combined Rietveld and Stereochemical Restraint Refinement of a Protein Crystal Structure. J. Appl. Crystallogr. 32:1084-1089.

Wilkinson, C. and Lehmann, M.S. 1991. Quasi-Laue Neutron Diffractometer. Nucl. Instr. Meth. Phys. Res. A310:411-415. 
Wilson, C.C. 1999. Single Crystal Neutron Diffraction from Molecular Materials. World Scientific, Singapore, New Jersey, London, Hong Kong.

Yuan, H.S.H., Stevens, R.C., Bau, R., Mosher, H.S., and Koetzle, T.F. 1994.

Determination of the Molecular Configuration of (+)-Neopentyl-1-d Alcohol by

Neutron and X-ray Diffraction Analysis. Proc. Natl. Acad. Sci. USA 91:12872-12876.

\section{KEY REFERENCES}

Bacon, G.E. 1975. Neutron Diffraction (Third Edition). Oxford University Press, London.

A classic treatment of the principles and practice of neutron diffraction. Chapter 4 is devoted to a description of experimental techniques.

Marshall, G.W., and Lovesey, S.W. 1971. Theory of Thermal Neutron Scattering. Oxford University Press, London.

Exhaustive treatment of all aspects of neutron scattering, including inelastic and quasielastic scattering, as well as diffraction. Sometimes referred to as "the bible" of neutron scattering. 
Stout, G.H. and Jensen, L.H. 1968. X-ray Structure Determination: A Practical Guide. Macmillan, New York, Toronto.

An excellent basic crystallography text outlining the techniques used in crystal structure analysis.

Willis, B.T.M. and Pryor, A.W. 1975. Thermal Vibrations in Crystallography. Cambridge University Press.

Provides a comprehensive overview of the use of crystallographic techniques to analyze thermal motion and disorder in solids.

Wilson, C.C. 1999. Single Crystal Neutron Diffraction from Molecular Materials. World Scientific, Singapore, New Jersey, London, Hong Kong.

A compendium describing the current state-of-the-art and giving a review of the literature including many recent results in neutron crystal structure analysis.

\section{FIGURE CAPTIONS}

Figure 1. Neutron scattering amplitudes as a function of atomic weight. Adapted from Figure 22 of Bacon (1975). 
Figure 2. The molecular structure of a tin(II) hydride complex determined at $20 \mathrm{~K}$ (Henning et al., 2000). Estimated standard deviations in bond distances and angles are less than $0.01 \AA$ and $0.1^{\circ}$, respectively.

Figure 3. Contoured neutron Fourier map for (+)-neopentyl-1-d-alcohol (S) with positive nuclear scattering density at $\mathrm{C}$ and $\mathrm{D}$ shown as black contours and negative nuclear scattering density at $\mathrm{H}$ shown as red contours (Yuan et al., 1994).

Figure 4. Anharmonicity of atomic displacements in zincblende as a function of temperature (Moss, McMullan and Koetzle, 1980; Moss, Roberts, McMullan and Koetzle, 1983).

Figure 5. Schematic plan of the SCD spectrometers formerly installed at the dual thermal beam port $\mathrm{H} 6$ at the Brookhaven HFBR.

Figure 6. Schematic plan of the SCD spectrometer installed on a liquid methane moderator at the Argonne IPNS. 


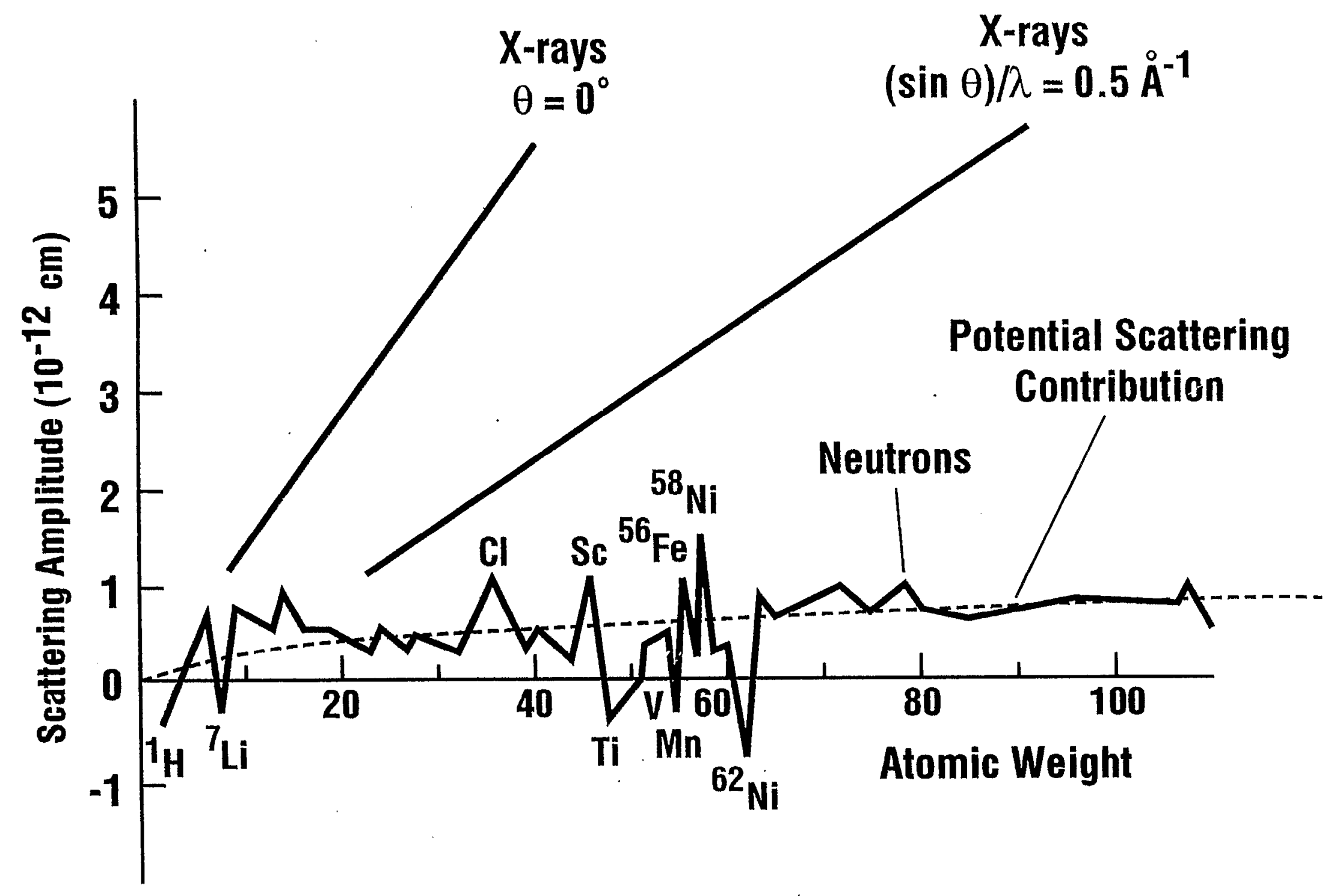




\section{Neutron Diffraction Study of Tin(II) Hydride at $20 \mathrm{~K}$ on IPNS SCD}

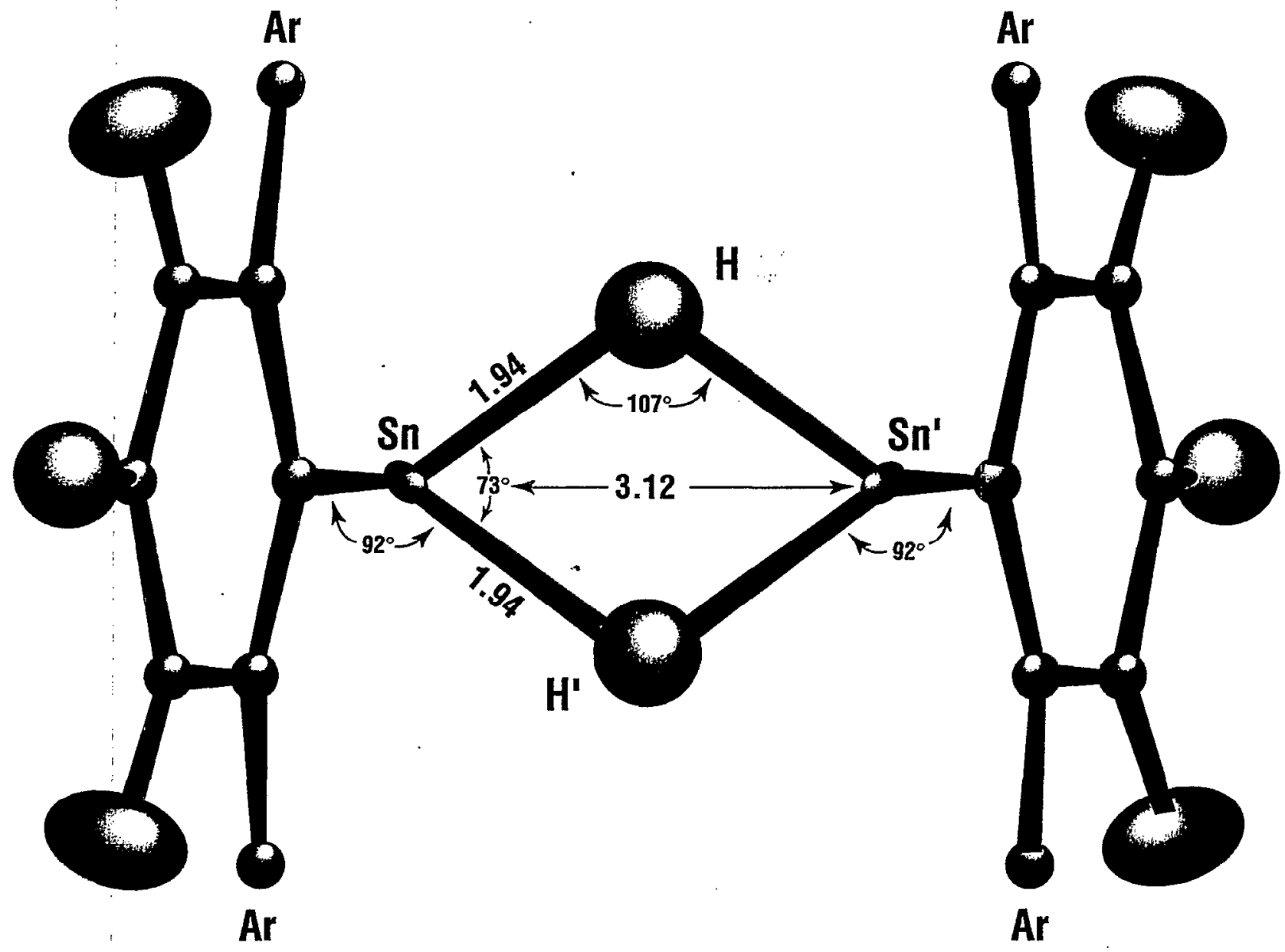

Henning, Albinati, Klooster, Koetzle, Power and Schultz (2000) 


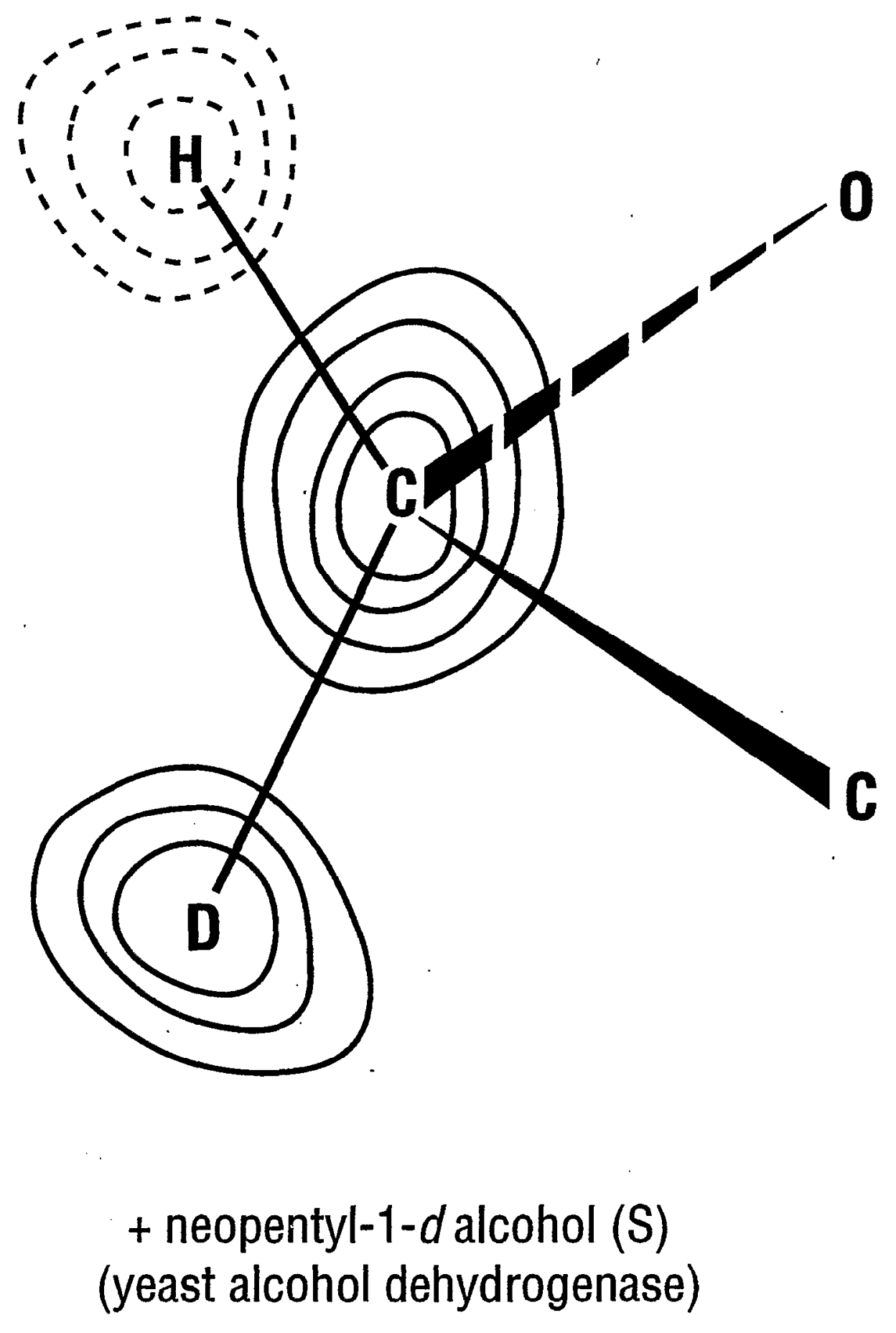




\section{Anharmonicity of Atomic Displacements}

\section{ZnS (m.p. 1973 K) - Cubic Phase}

\section{Anharmonicity}
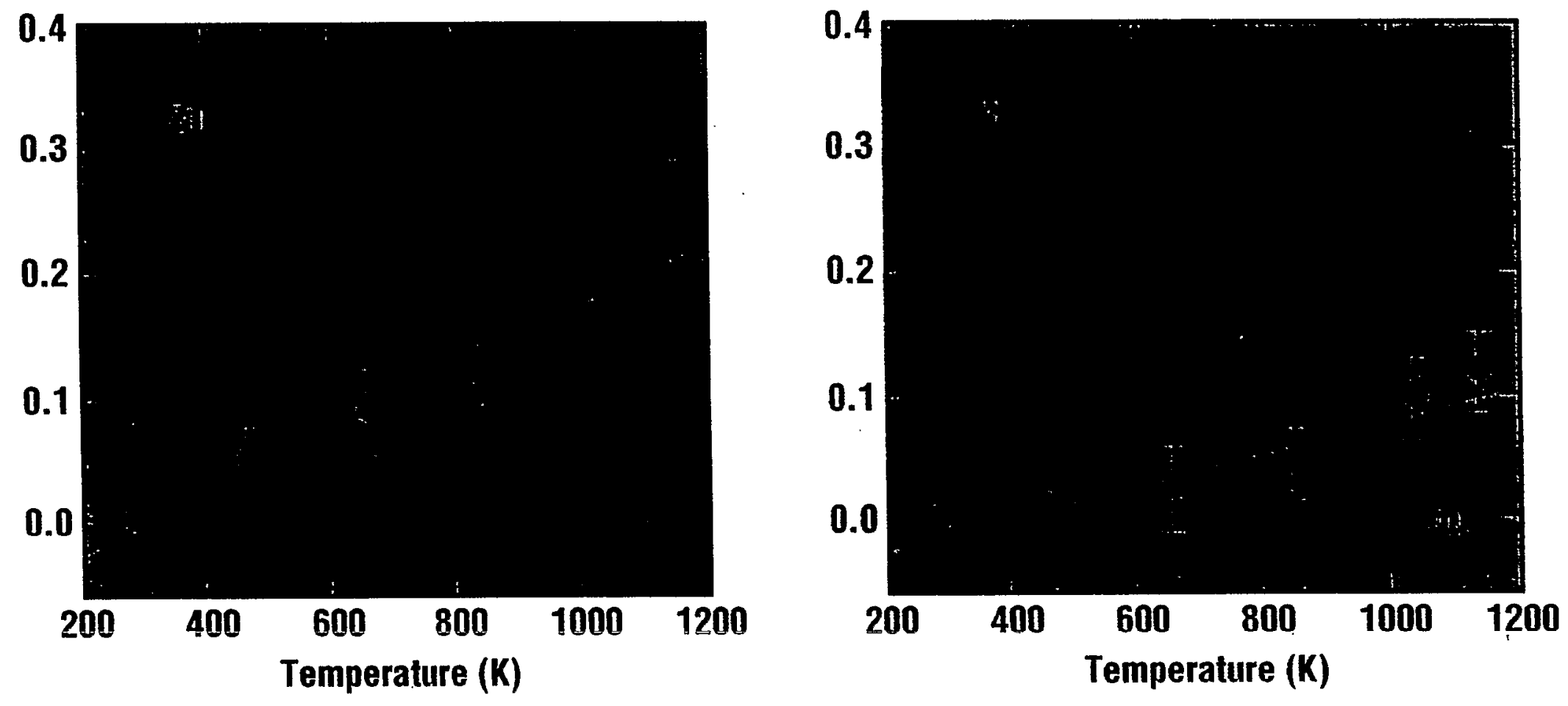

Moss, McMullan and Koetzle (1980) 


\section{Brookhaven High Flux Beam Reactor (HFBR) Schematic Plan of Spectrometers Installed at $\mathrm{HG}$}

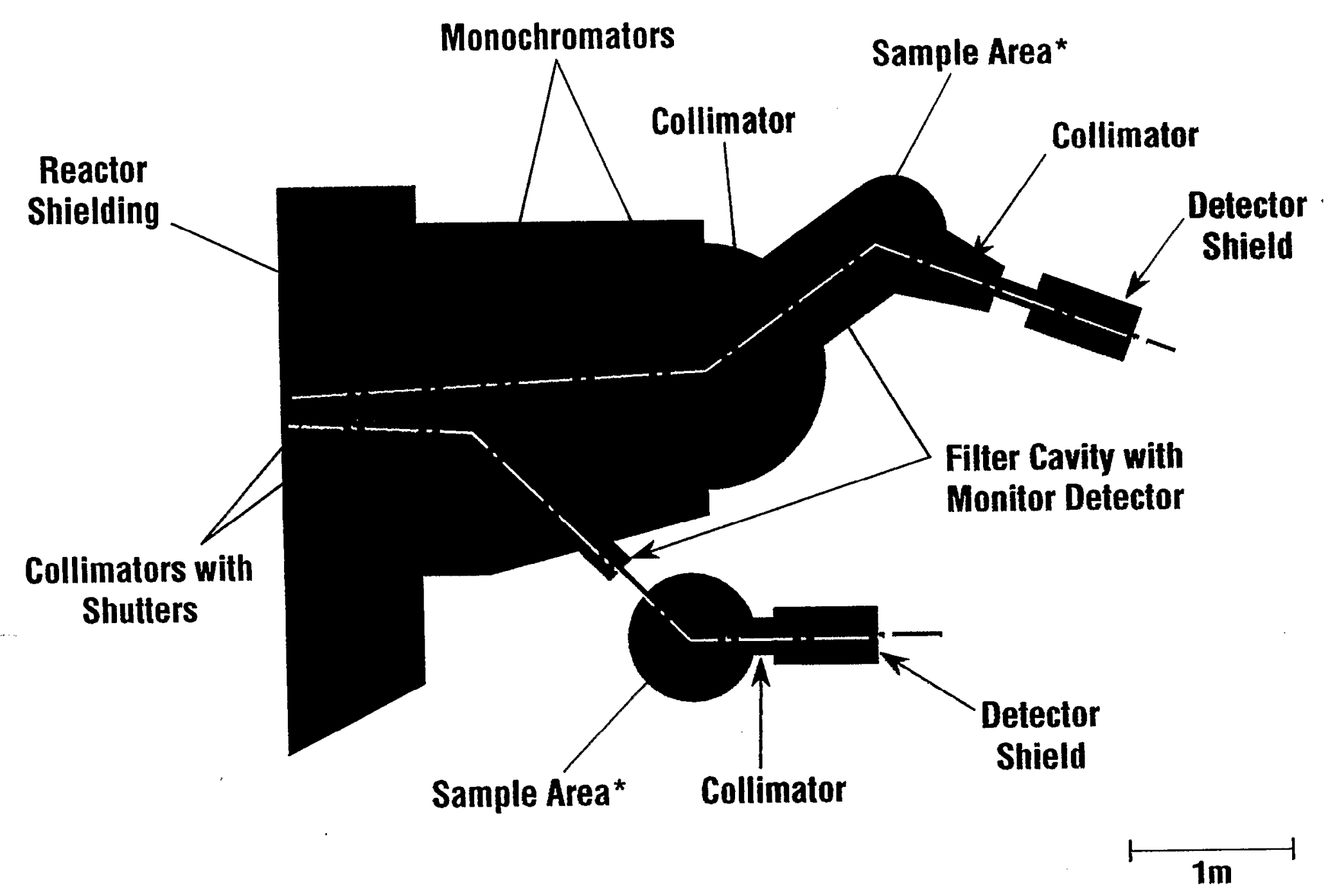

* Location of a 4-circle diffractometer with optional cryostat mounted on $\chi$-circle. 


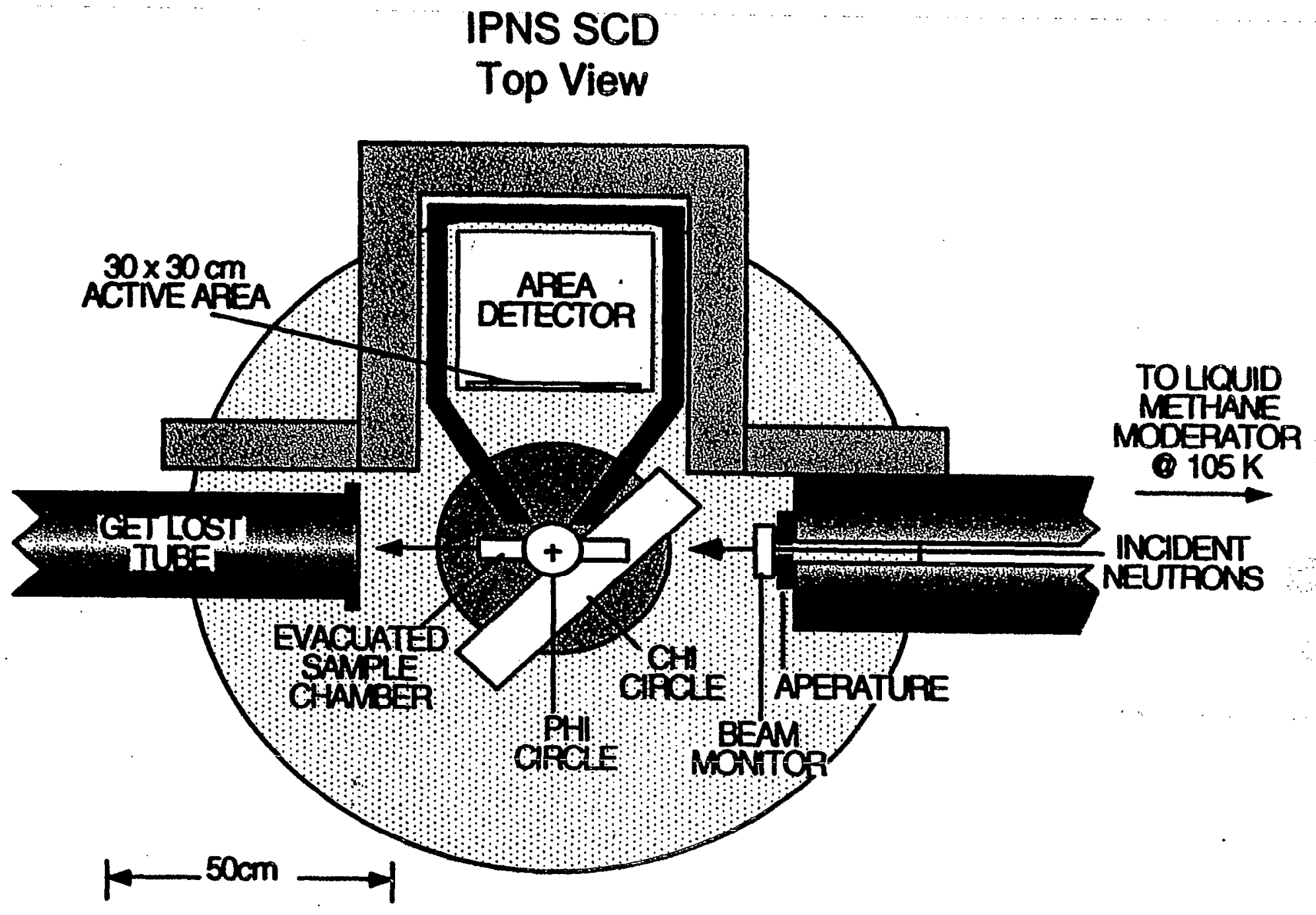




\section{APPENDIX}

Table 1. Neutron SCD Instruments and Facility Website Contacts.

\begin{tabular}{|c|c|c|c|c|c|c|}
\hline Facility & Source Type & Location & $\begin{array}{l}\text { Instrument } \\
\text { Name }\end{array}$ & $\begin{array}{l}\text { Instrument } \\
\text { Characteristics }\end{array}$ & Proposal Cycle & Website Url \\
\hline BENSC & Reactor & $\begin{array}{l}\text { Hahn-Meitner Institute, } \\
\text { Berlin, Germany }\end{array}$ & E5 & Monochromatic thermal beam & Semiannual & www.hmi.de/bensc \\
\hline$\overline{\text { DR3 }}$ & Reactor & $\begin{array}{l}\text { Risø National Laboratory, } \\
\text { Roskilde, Denmark }\end{array}$ & & Monochromatic thermal beam & Every 4 months ${ }^{\ddagger}$ & $\begin{array}{l}\text { www. fys.risoe.dk/fys/rom/neutr } \\
\text { ons/userprog/tmrintro.html }\end{array}$ \\
\hline FRJ-2 & Reactor & $\begin{array}{l}\text { Jülich Research Center, } \\
\text { Jülich, Germany }\end{array}$ & SV28 & Monochromatic thermal beam & Consult web site & $\begin{array}{l}\text { www.kfa.juelich.de/iff/institute/ } \\
\text { ins/Broschuere NSE }\end{array}$ \\
\hline HIFAR $^{\S}$ & Reactor & $\begin{array}{l}\text { ANSTO, Lucas Heights, } \\
\text { NSW, Australia }\end{array}$ & 2TANA & Monochromatic thermal beam & Consult web site & www.ansto.gov.au \\
\hline HFIR & Reactor & $\begin{array}{l}\text { Oak Ridge National } \\
\text { Laboratory, Oak Ridge, } \\
\text { TN, USA }\end{array}$ & HB-2A & Monochromatic thermal beam & Continuous & www.ornl.gov/hfir \\
\hline \multirow[t]{4}{*}{ HFR } & Reactor & $\begin{array}{l}\text { Institut Laue-Langevin, } \\
\text { Grenoble, France }\end{array}$ & D9 & Monochromatic hot beam & Semiannual & www.il.fr \\
\hline & & & D10 & $\begin{array}{l}\text { Monochromatic 4-circle and } \\
\text { triple axis }\end{array}$ & & \\
\hline & & & D19 & Monochromatic thermal beam & & \\
\hline & & & LADI & $\begin{array}{l}\text { Laue with imaging plate. } \\
\text { Weissenberg geometry. }\end{array}$ & & \\
\hline
\end{tabular}

\footnotetext{
${ }^{\dagger}$ The instruments are 4-circle diffractometers unless otherwise noted.
}

${ }^{\ddagger} \mathrm{DR} 3$ and $\mathrm{R} 2$ operate a unified proposal system.

${ }^{\S}$ Construction is underway on a replacement for the HIFAR reactor and is scheduled for completion in 2005. 
APPENDIX

Table 1. Neutron SCD Instruments and Facility Website Contacts, ctd.

\begin{tabular}{|c|c|c|c|c|c|c|}
\hline Facility & $\begin{array}{l}\text { Source } \\
\text { Type }\end{array}$ & Location & $\begin{array}{l}\text { Instrument } \\
\text { Name }\end{array}$ & $\begin{array}{l}\text { Instrument } \\
\text { Characteristics }^{\dagger}\end{array}$ & Proposal Cycle & Website Url \\
\hline IBR-2 & Reactor & $\begin{array}{l}\text { Franck Laboratory of } \\
\text { Nuclear Physics, Dubna, } \\
\text { Russia }\end{array}$ & DN2 & Monochromatic thermal beam & Consult web site & www.nfdfn.jinr.dubna.su \\
\hline IPNS & Spallation & $\begin{array}{l}\text { Argonne National } \\
\text { Laboratory, Argonne, IL, } \\
\text { USA }\end{array}$ & SCD & Time-of-flight Laue & Semiannual & www.pus.anl.gov \\
\hline ISIS & Spallation & $\begin{array}{l}\text { Rutherford Appleton } \\
\text { Laboratory, Chilton, } \\
\text { Oxon, UK }\end{array}$ & SXD & Time-of-flight Laue & Semiannual & $\frac{\text { www.nd.rl.ac.uk }}{.}$ \\
\hline JRR-3 & Reactor & $\begin{array}{l}\text { Japan Atomic Energy } \\
\text { Research Institute, Tokai- } \\
\text { mura, Japan }\end{array}$ & BIX-III & $\begin{array}{l}\text { Monochromatic thermal beam } \\
\text { with imaging plate. Oscillation } \\
\text { geometry. }\end{array}$ & Consult web site & www.jaeri.go.jp \\
\hline$\overline{\text { KENS }}$ & Spallation & $\begin{array}{l}\text { KEK Laboratory, } \\
\text { Tsukuba, Japan }\end{array}$ & FOX & Time-of-flight Laue & Annual & http://neutron-www.kek.jp \\
\hline LANSCE & Spallation & $\begin{array}{l}\text { Los Alamos National } \\
\text { Laboratory, Los Alamos, } \\
\text { NM, USA }\end{array}$ & SCD & Time-of-flight Laue & Consult web site & www.lansce.lanl.gov \\
\hline NRU & Reactor & $\begin{array}{l}\text { Chalk River Laboratories, } \\
\text { Chalk River, Ontario, } \\
\text { Canada }\end{array}$ & E3 & Monochromatic thermal beam & Continuous & http://neutron.nrc.ca \\
\hline Orphée & Reactor & $\begin{array}{l}\text { Laboratory Léon } \\
\text { Brillouin, Saclay, France }\end{array}$ & $5 \mathrm{C} 2$ & Monochromatic hot beam & Semiannual & http://www-llb.cea.fr \\
\hline
\end{tabular}

\footnotetext{
${ }^{\dagger}$ The instruments are 4-circle diffractometers unless otherwise noted.
} 


\section{APPENDIX}

Table 1. Neutron SCD Instruments and Facility Website Contacts, ctd.

\begin{tabular}{|c|c|c|c|c|c|c|}
\hline Facility & $\begin{array}{l}\text { Source } \\
\text { Type }\end{array}$ & Location & $\begin{array}{l}\text { Instrument } \\
\text { Name }\end{array}$ & $\begin{array}{l}\text { Instrument } \\
\text { Characteristics }^{\dagger}\end{array}$ & Proposal Cycle & Website Url \\
\hline $\mathrm{R} 2$ & Reactor & $\begin{array}{l}\text { Studsvik Neutron } \\
\text { Research Laboratory, } \\
\text { Nykobing, Sweden }\end{array}$ & SXD & Monochromatic thermal beam & Every 4 months $^{\ddagger}$ & www.studsvik.uu.se \\
\hline SINQ & $\begin{array}{l}\text { CW } \\
\text { Spallation }\end{array}$ & $\begin{array}{l}\text { Paul Scherer Institute, } \\
\text { Villigen, Switzerland }\end{array}$ & TriCS & Monochromatic thermal beam & Semiannual & www.psi.ch \\
\hline
\end{tabular}

\footnotetext{
${ }^{\dagger}$ The instruments are 4-circle diffractometers unless otherwise noted.

${ }^{\ddagger} \mathrm{DR} 3$ and R2 operate a unified proposal system.
} 